\title{
Empoderamiento enfermero en las unidades de cuidados intensivos de adultos en el Hospital Central del Instituto de Previsión Social
}

\author{
Nancy Villagra Rivera, *Mónica Ruoti Cosp \\ Universidad Católica "Nuestra Señora de la Asunción". Paraguay \\ Cómo referenciar este artículo/ \\ How to reference this article: \\ Villagra Rivera N, Ruoti Cosp M. Empoderamiento \\ enfermero en las unidades de cuidados intensivos de \\ adultos en el Hospital Central del Instituto de Previsión \\ Social. Mem. Inst. Investig. Cienc. Salud. 2018; \\ 16(1):84-93
}

\section{R E S U M E N}

El empoderamiento es el proceso por el cual las personas fortalecen sus capacidades, confianza, visión y protagonismo para impulsar cambios positivos de las situaciones que viven, por lo que es un desafío para los profesionales de enfermería en todos los niveles de asistencia. El objetivo de esta investigación es realizar un análisis del empoderamiento de los enfermeros asistenciales de cuidados intensivos de adultos en el Hospital Central del Instituto de Previsión Social en la ciudad de Asunción, Paraguay. Se realizó un estudio cualitativo con enfoque fenomenológico. Los datos se recolectaron a través de entrevistas semi estructuradas grabadas cuyas preguntas se fundamentaron en seis categorías de estudio: sentido de pertenencia, autonomía, liderazgo, trabajo en equipo, participación en asociaciones y percepción de empoderamiento. La muestra se determinó por conveniencia y saturación alcanzando a 17 sujetos. Las respuestas de los participantes se agruparon en ideas comunes para deducir los resultados. En el análisis se tuvo en cuenta las propuestas de Zimmerman y Benner en materia de empoderamiento y desarrollo profesional de enfermería respectivamente. En el grupo estudiado se distinguen atributos como autonomía y trabajo en equipo que favorecen el empoderamiento individual. Sin embargo, estas cualidades contrastan con un débil sentido de pertenencia, una práctica relativa del liderazgo y escasa participación en asociaciones. En consecuencia, el empoderamiento de enfermería en las unidades de cuidados intensivos estudiadas es un proceso incipiente que requiere intervenciones efectivas para consolidarse y fortalecer la identidad profesional.

Palabras clave: empoderamiento, enfermero/a asistencial, cuidados intensivos.

\section{Nursing empowerment in the adult intensive care units at the Central Hospital of the Instituto de Previsión Social}

\section{A B S T R A C T}

Empowerment is a process by which people strengthen their abilities, confidence, vision and roles to promote positive changes in the situations they go through, therefore it is a challenge for nursing professionals at all levels of assistance. The objective of this study was to analyze the nurse empowerment at the adult intensive care of the Hospital Central del Instituto de Previsión Social in Asunción Paraguay. A qualitative study with a phenomenological approach was carried out. Data was collected through recorded semistructured interviews with questions based on six categories of study: sense of belonging, autonomy, leadership, teamwork, participation in associations and perception of empowerment. A convenience sampling reaching saturation with 17 subjects was used. Answers of the participants were grouped into common ideas to deduce the results. Analysis took into account the Zimmerman and Benner proposals in terms of empowerment and professional nursing development, respectively. In the studied group, attributes such as autonomy and teamwork were distinguished as favoring individual empowerment. However,

Fecha de recepción: febrero 2018. Fecha de aceptación: marzo 2018

*Autor correspondiente: Mónica Ruoti Cosp. Universidad Católica "Nuestra Señora de la Asunción". Paraguay Email: monicaruoti@gmail.com 
these qualities contrast with a weak sense of belonging, a relative practice of leadership and little participation in associations. Consequently, the nursing empowerment in the intensive care units studied is an incipient process that requires effective interventions to consolidate and strengthen the professional identity.

Keywords: empowerment, nursing care, intensive care.

\section{INTRODUCCIÓN}

Dentro de las instituciones de salud, enfermería ocupa un lugar sobresaliente, en primer lugar porque supera en número a los demás profesionales que conforman las organizaciones y en segundo lugar, porque gracias a sus intervenciones se logran en gran parte los objetivos terapéuticos propuestos por el equipo sanitario.

En las unidades de cuidados intensivos, la condición del enfermo crítico requiere atención, vigilancia y contención las 24 horas del día y quien dispensa este servicio es el enfermero asistencial. Por este motivo, cada profesional enfermero/a se ocupa del cuidado de uno, dos y en algunos excepcionales casos, tres pacientes en cada guardia. Por otra parte, la familia y los demás integrantes del equipo de terapia intensiva, también convergen en un enfermero/a para conocer la evolución del paciente.

Pese a ser importantes en la evolución de los enfermos, los profesionales de enfermería muestran un perfil bajo en relación a otros miembros del equipo. Sus acciones son silenciosas, carecen de evidencia y principalmente, muestran desorganización individual y colectiva.

Ante esta realidad, nace la inquietud por analizar el empoderamiento profesional en los enfermeros asistenciales que trabajan en las áreas críticas del Hospital Central del Instituto de Previsión Social (IPS). La respuesta se busca en los propios enfermeros, quienes a través de sus vivencias cotidianas muestran lo que son y lo que hacen.

El empoderamiento se ha definido como "el proceso por el cual las personas fortalecen sus capacidades, confianza, visión y protagonismo como grupo social para impulsar cambios positivos de las situaciones que viven"(1).

La propuesta de Marc Zimmerman en materia de empoderamiento(2) y Patricia Benner en lo relacionado el desarrollo profesional de enfermería ${ }^{(3)}$, constituyen el enfoque teórico desde donde se aborda la problemática.

\section{MATERIALES Y MÉTODO}

Para describir la problemática con mayor profundidad, este estudio tiene un abordaje cualitativo, con enfoque fenomenológico que utiliza los relatos de los participantes aprovechando al máximo la riqueza de las experiencias de las personas ${ }^{(4)}$.

El estudio fue aplicado a los profesionales que trabajan como enfermeros asistenciales en los servicios dependientes del Departamento de Medicina Crítica del Hospital Central del IPS (Unidad de Terapia Intensiva de Adultos, de Terapia Intermedia y Unidad Coronaria), ubicado en la ciudad de Asunción, Paraguay. Se entrevistaron en total a 17 profesionales.

Se ha seleccionado la muestra por conveniencia y saturación de la información, teniendo en cuenta a enfermeros/as asistenciales, con y sin estudios de post grado, contratados/as y nombrados/as, de cualquier turno y que aceptaron participar del estudio.

Se realizaron entrevistas semi estructuradas en base a una guía de preguntas abiertas. La guía primaria contiene 28 preguntas que hacen referencia a las subcategorías de estudio. Es un instrumento flexible que permite improvisar nuevas preguntas de acuerdo a las respuestas conferidas por el participante.

Para la recolección de los datos se solicitó un permiso por escrito a las autoridades de la institución, a las jefas de enfermería y jefes médicos de los servicios. Las solicitudes estuvieron acompañadas del protocolo de investigación aprobado previamente por el departamento de educación y el comité de ética del Hospital Central del IPS.

Luego de la inmersión total en el ambiente de estudio, se contactó a los enfermeros asistenciales a través de las jefas de enfermería y responsables de cada turno. Fueron invitados a participar todos los aquellos que cumplían con los criterios de inclusión. Con quienes aceptaron colaborar, se programó una cita para llevar a cabo la entrevista.

Durante las entrevistas, se entregó a cada participante un consentimiento informado explicando el objetivo del estudio, éste debía ser firmado y devuelto. Un duplicado del mismo quedó en poder de cada participante. También debieron completar sus datos socio 
demográfico en un pequeño formulario. En este momento se asignó al entrevistado un código numérico de dos cifras para identificarlo durante el estudio.

Las entrevistas fueron almacenadas en una grabadora digital y se desarrollaron, de manera flexible, de acuerdo a una guía que permitió indagar los puntos considerados en las categorías de estudio además de otros que resultaron del discurso del entrevistado. Al realizar las entrevistas a los profesionales de enfermería, se tuvo en cuenta la multi culturalidad de la población paraguaya, así como su bilingüismo.

En forma confidencial se transcribieron la totalidad de las entrevistas y luego, en una segunda etapa, fueron mostradas a los respectivos entrevistados para su aprobación. Esta aprobación consistió en la impronta de una media firma en cada hoja de la entrevista.

A partir de la revisión bibliográfica se identificaron cinco cualidades que posee la persona empoderada que constituyeron las categorías de estudio respecto al empoderamiento. Esquema 1.

En forma empírica, se crearon las siguientes sub categorías: para el sentido de pertenencia: relacionamiento, integración convivencia y satisfacción laboral; para la autonomía: libertad y competencia profesional: para el liderazgo: responsabilidad e influencia positiva; para el trabajo en equipo: percepción de trabajo en equipo, solidaridad, comunicación, respeto y tolerancia.

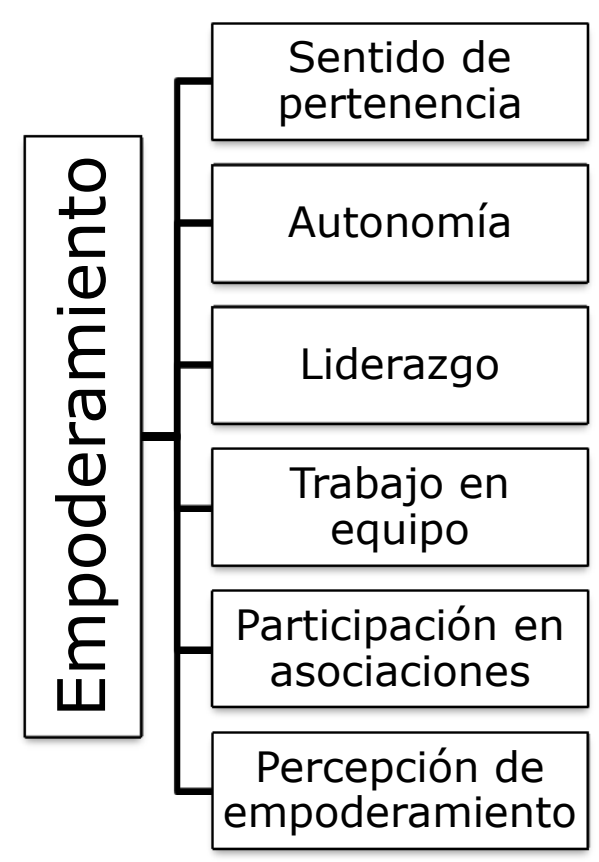

Esquema 1. Categorías de estudio del empoderamiento

\section{RESULTADOS}

La caracterización socio-demográfica de los 17 entrevistados sujetos de estudio se presenta en la Tabla 1. La mayoría de los participantes del estudio era del sexo femenino $(n=12)$ con un promedio de edad de 34 años, promedio en la profesión de 10 años, promedio de tiempo que trabaja en IPS de 8 años y en el servicio 7 años. En ocho el mayor grado académico logrado era de especialista, dos de maestría y el resto licenciatura. El número de vínculos laborales fue en la gran mayoría más de uno; dos vínculos en 10 y tres vínculos en cuatro. 
Tabla 1. Características socio demográficas de los enfermeros/as asistenciales que trabajan en los servicios de medicina crítica Hospital Central IPS, Asunción, Paraguay 2015.

\begin{tabular}{|c|c|c|c|c|c|c|c|c|}
\hline Código & Sexo & Edad & $\begin{array}{l}\text { Años de } \\
\text { ejercicios } \\
\text { de la } \\
\text { profesión }\end{array}$ & $\begin{array}{l}\text { Tiempo } \\
\text { que } \\
\text { trabaja } \\
\text { en IPS }\end{array}$ & $\begin{array}{l}\text { Tiempo } \\
\text { que } \\
\text { trabaja } \\
\text { en el } \\
\text { servicio }\end{array}$ & $\begin{array}{l}\text { Mayor grado } \\
\text { académico }\end{array}$ & $\begin{array}{l}\text { Número } \\
\text { de } \\
\text { vínculos } \\
\text { laborales }\end{array}$ & $\begin{array}{l}\text { Turno en el } \\
\text { cual trabaja } \\
\text { en IPS }\end{array}$ \\
\hline 01 & $\mathrm{~F}$ & 37 & 7 & 7 & 7 & Especialista & 2 & FSD \\
\hline 02 & $\mathrm{~F}$ & 34 & 8 & 6 & 6 & Especialista & 2 & Noche \\
\hline 03 & $M$ & 34 & 7 & 7 & 7 & Especialista & 3 & Noche \\
\hline 04 & $\mathrm{~F}$ & 35 & 8 & 7 & 7 & Especialista & 3 & Noche \\
\hline 05 & $\mathrm{~F}$ & 34 & 13 & 13 & 13 & Licenciatura & 1 & Tarde \\
\hline 06 & $\mathrm{~F}$ & 33 & 9 & 8 & 5 & Licenciatura & 2 & Tarde \\
\hline 07 & $\mathrm{~F}$ & 33 & 12 & 12 & 10 & Especialista & 2 & Mañana \\
\hline 08 & $\mathrm{~F}$ & 45 & 24 & 8 & 8 & Especialista & 2 & Noche \\
\hline 09 & $\mathrm{~F}$ & 36 & 14 & 5 & 5 & Licenciatura & 2 & FSD \\
\hline 10 & $\mathrm{~F}$ & 37 & 12 & 8 & 8 & Maestría & 2 & FSD \\
\hline 11 & $\mathrm{~F}$ & 34 & 14 & 8 & 8 & Especialista & 1 & FSD \\
\hline 12 & $M$ & 29 & 5 & 5 & 5 & Licenciatura & 1 & Noche \\
\hline 13 & M & 35 & 12 & 11 & 5 & Maestría & 3 & Noche \\
\hline 14 & $\mathrm{~F}$ & 28 & 11 & 7 & 6 & Licenciatura & 2 & Noche \\
\hline 15 & $\mathrm{~F}$ & 35 & 11 & 9 & 9 & Especialista & 3 & Noche \\
\hline 16 & M & 34 & 7 & 5 & 5 & Licenciatura & 2 & FSD \\
\hline 17 & $M$ & 27 & 4 & 4 & 4 & Licenciatura & 2 & FSD \\
\hline
\end{tabular}

Los resultados obtenidos en cada categoría de estudio se indican mostrando las ideas recogidas en cada subcategoría.

\section{Sentido de Pertenencia}

El relacionamiento entre compañeros se manifiesta como bueno cuando los entrevistados refieren que se sienten a gusto, incluidos/as y sin problemas entre ellos. Por el contrario, la mala relación tiene que ver con los conflictos existentes y el incumplimiento de las reglas de la institución. Se observa, que los problemas no resueltos, afectan además la convivencia de los enfermeros asistenciales.

En cuanto a la integración, ésta se percibe como existente o ausente. Hay referencias a la pérdida de la integración, lo que hace suponer que en algún momento estuvieron integrados y que por diversos motivos ahora no lo están. Esta situación genera incomodidad y afecta negativamente la convivencia entre los enfermeros asistenciales.

Los participantes manifiestan que la satisfacción en el trabajo tiene que ver con la posibilidad de sentirse útil, el acostumbramiento y conocimiento del área. Por otro lado, la insatisfacción se relaciona con el crecimiento personal, el cual supone que la etapa de enfermero asistencial es para quienes se inician en la enfermería y que una vez superada esa etapa, se debe avanzar hacia niveles gerenciales dentro de la estructura sanitaria.

Los enfermeros/as encuentran múltiples obstáculos para el crecimiento profesional, los cuales van desde problemas familiares, múltiples empleos que dificultan tomarse tiempo para estudiar, falta de apoyo institucional para facilitar la capacitación y falta de recursos económicos. A pesar de estos obstáculos, los entrevistados reconocen la importancia de capacitarse en forma continua y se muestran de acuerdo en que la capacitación tiene que ver con el interés personal de superarse.

Otro aspecto importante hallado en esta categoría, es el hecho que los participantes aseguran que trabajando en las unidades de cuidados intensivos, siempre están aprendiendo algo; que la práctica les da la posibilidad de ir ganando experiencia y acumulando conocimientos.

El sentido de pertenencia hacia la profesión es una motivación aún incipiente entre los profesionales de enfermería que trabajan en las áreas críticas. 


\section{Autonomía}

En relación a la sub categoría libertad, los profesionales de enfermería afirman que la mayor parte del tiempo deben tomar decisiones relacionadas con el cuidado del paciente y por el bien del paciente. Algunos entrevistados afirman que las decisiones que toman, no son tenidas en cuenta por los demás integrantes del equipo asistencial, especialmente los médicos.

Los participantes aseguran que se sienten capaces de tomar decisiones y que esta capacidad la adquieren gracias a la experiencia y el conocimiento adquiridos en la práctica clínica. Reconocen que la autonomía se refiere a la capacidad de tomar decisiones y está relacionada a la competencia profesional. Es una práctica habitual que implica priorizar acciones de cuidado al enfermo y se fundamenta en el hecho de que el profesional de enfermería es quien permanece la mayor parte del tiempo junto al paciente crítico y por lo tanto, lo conoce mejor.

Se evidencia además, que la falta de autonomía tiene relación con la dependencia médica y la percepción de que la enfermería corresponde a un rango inferior al médico en la jerarquía institucional.

El analizar ambas subcategorías, se puede afirmar que la autonomía es una práctica sistemática entre los profesionales de esta área.

\section{Liderazgo}

La responsabilidad en el ejercicio del liderazgo se indagó a través del reconocimiento de la función que cumplen en el servicio. A este respecto, todos los entrevistados reconocen que son licenciados en enfermería en el área asistencial.

Estoy como enfermera asistencial, o sea que lo que hago es el cuidado directo a paciente; atención directa al paciente [E14].

Se identifican además, múltiples actividades dentro del rol asistencial, destacándose la educación e información a los familiares y la gestión de enfermería en lo relacionado a provisión de insumos, equipos y distribución de personal. Sólo un entrevistado limita la función asistencial al cumplimiento de las indicaciones médicas.

Los enfermeros asistenciales de las unidades de cuidados intensivos, revelan que la falta de insumos o materiales de trabajo, es la principal y más importante limitación para desempeñar sus funciones. Esta situación se considera nueva dentro del esquema de trabajo, lo que hace suponer que en algún momento se contó con los materiales necesarios, pero actualmente, no.

En relación a la influencia positiva, algunos entrevistados responden que no siempre es posible sugerir a un compañero alguna acción y que en todo caso depende del receptor.

Varias veces e incluso a veces me llaman a preguntar cómo era, cómo es que dijiste la vez pasada, qué hiciste, cómo es que se hace. Entonces ahí vos te das cuenta que tienen en cuenta lo que vos les sugieres. Inclusive los médicos a veces aceptan nuestras sugerencias [E9].

Por ejemplo, hoy pasó emm, el paciente estaba muy delicado, verdad, y yo le dije, compañera una sugerencia; por qué no le ponemos derecha y esperamos que se estabilice $y$ después le damos la vuelta otra vez, porque la señora era muy grande. Y me hizo caso y había sido su tubo estaba por salir y ya me había pasado la otra vez [E11].

Sí... sí, habitualmente entre varones no hay luego problema. Pero entre mujeres ellas se chocan muchísimo [E16].

Otros consideran que las sugerencias que dan a sus compañeros de trabajo son bien acogidas ya que son parte del trabajo en equipo.

Un motivo importante para que los compañeros no tomen en cuenta las sugerencias, es el hecho de que las reglas no son claras y esto hace que el liderazgo sea difícil de practicar.

Por lo tanto, la práctica del liderazgo en los enfermeros asistenciales estudiados, es una práctica condicionada, ya que existen factores negativos que impiden su total ejercicio.

\section{Trabajo en equipo}

Los enfermeros asistenciales perciben que trabajan en equipo dentro de su guardia y que esta forma de trabajo es propia de las unidades de cuidados intensivos.

Sí, sí. Yo creo que la terapia intensiva es uno de los lugares donde... yo creo que más se ve el trabajo en equipo, sí. No precisamente quizás porque esté a gusto o no, si no porque 
hay esa necesidad, o sea el paciente crítico necesita, requiere del trabajo en equipo. Tanto el médico no puede hacer solo muchas cosas, ni la enfermera, ni el fisioterapeuta; todos nos necesitamos [...] [E15].

Algunos entrevistados afirman que se ha perdido la posibilidad de trabajar en equipo debido a problemas como falta de entendimiento y armonía dentro del grupo.

No, acá en el servicio no. No se trabaja en equipo. Cada quien hace por su cuenta lo que mejor le parece. La toma de decisiones no es compartida. [...] cada quien toma sus decisiones y cada quien tiene su modo de actuar y cada quien tiene sus propios protocolos y evidentemente no hay congruencia entre uno y otro [E13].

La acción de ser solidario implica no tener que pedir ayuda para compartir las tareas; está relacionada con el compañerismo y la armonía del grupo.

Entre las acciones que demuestran solidaridad por parte de los entrevistados se identifican la colaboración en los procedimientos de enfermería tales como higiene, movilización de los pacientes, apoyo en la reanimación, preparación de drogas y administración de medicamentos.

[...] acá todos nos necesitamos todos, entonces no hay mucho para pensar, verdad [E15].

Una cualidad interesante detectada en esta sub categoría, es la falta de vocación.

[...] muchos ya vienen para cobrar no más [...] [E4].

La comunicación es percibida como buena cuando existe empatía, cuando se resuelven los conflictos conversando con el grupo o con el médico y cuando existe sinceridad para decir las cosas que molestan. Es importante destacar que el uso de la tecnología, se identifica como un factor que favorece la buena comunicación entre los integrantes del equipo de trabajo.

[...] la tecnología hace que nos comuniquemos cada vez mejor y por decirte yo ya sé qué tipo de pacientes voy a tener esta noche porque ya me lo pasaron por el WhatsApp [...] [E8].

Cuando se percibe que hay una mala comunicación, ésta se relaciona con los conflictos no resueltos entre compañeros y falta de fluidez en las directrices.

Del uno al cinco... dos. Y es como un teléfono cortado... No es muy fluida. La información va para dos o tres personas y no llega a todas [E11].

Se puede afirmar que el respeto está vigente y se da en forma recíproca entre compañeros del servicio: dar para recibir. Los enfermeros asistenciales se consideran iguales entre ellos y manifiestan sus diferencias en un ambiente de sinceridad y buen diálogo. No obstante, se identifican hechos puntuales de compañeros exigentes e incluso discusiones entre los integrantes del grupo. Sin embargo, los participantes aseguran que las normativas del servicio y la institución, no están difundidas adecuadamente y si bien algunas normas son conocidas, no se respetan.

Las normas y reglamentos, conocemos cuando hay algo que nos preocupa nada más. No hay una buena difusión de las reglas ni las normas verdad, yo no encuentro [E1].

Supuestamente hay reglas que nadie le hace caso [E4].

Los entrevistados refieren que existe tolerancia en el grupo y en el servicio. Ser tolerante significa, no tener conflictos graves y aguantar el carácter de los compañeros. Cuando la tolerancia se termina, se inician los problemas.

El trabajo en equipo, se percibe como un valor importante para quienes se desempeñan como enfermeros asistenciales en las unidades de cuidados intensivos, ya que favorece el acto de cuidar. Se identifican conductas usuales en relación al trabajo en equipo que fortalecen al grupo y generan cohesión.

\section{Participación en las asociaciones}

Los enfermeros asistenciales identifican a la Asociación Paraguaya de Enfermería y los sindicatos de trabajadores dentro del Hospital Central. Manifiestan que se encuentran asociados, pero en general, la participación es pasiva y no se involucran activamente por 
motivos como falta de tiempo, desinterés y desconfianza, principalmente, hacia los líderes de las organizaciones. Hay quienes manifiestan que ingresaron a los sindicatos en forma obligatoria y que estar asociado a ellos implica un gasto de dinero.

Una participación obligatoria, He'i la tipa, en la cual no me preguntaron querés estar en este sindicato? [...] nunca. Que yo entré y ya apareció no más el descuento ahí, que no me genera ningún tipo de beneficio, más bien un gasto porque tenés que estar aportando algo que no pediste aportar [E3]. Es una participación pasiva, ahí no más, muy poca integración hay de mi parte, pero tal vez porque no nos dan la apertura que nosotros necesitamos [E1].

Otra corriente de opiniones muestra que los enfermeros asistenciales se asocian a los sindicatos por la necesidad de conocer sus derechos y beneficios.

En cuanto a la Asociación Paraguaya de Enfermería, los participantes manifiestan escaso interés por participar en ella. Incluso hay quienes afirman que la siguen sólo por redes sociales.

A la APE la sigo por el Facebook. No, no soy socia. [...] Solamente les sigo por el Facebook para ver en qué andan [E6].

No, ninguno, yo renuncié a toditos los sindicatos [...] nunca estuve en la $A P E$, nunca. Yo la verdad es que no le veo ningún beneficio, sinceramente. Yo no más no veo. No sé para otro tendrá otro punto de vista, pero yo no. Yo no personalmente [E16].

Y... Por necesidad estoy en la APE. No me gusta su sistema de trabajo, pero por necesidad estoy en la APE [...] [E13].

Sin embargo, reconocen que estas estructuras son necesarias cuando se pretende conseguir beneficios.

[...] Estoy asociada por cualquier eventualidad, cualquier problema [...] [E11].

Porque uno necesita de ese tipo de organizaciones para poder... Para que no lo estén rebasando en muchos derechos verdad [E15].

A partir de estos relatos, se puede afirmar que la adhesión a las organizaciones existentes en el medio no es considerada importante para el grupo estudiado y la participación en ellas tampoco es una prioridad.

\section{Percepción de empoderamiento}

Se indagó a través de la pregunta: ¿consideras que como profesional de enfermería estás empoderado?. Las respuestas son variadas y en algunos casos fue necesario aclaración o delimitación del concepto.

Hay quienes comprenden el significado de empoderamiento y manifiestan categóricamente que sienten empoderamiento individual.

Otro tipo de respuestas muestra que los enfermeros asistenciales confunden empoderamiento con liderazgo y participación.

Los entrevistados reconocen que la enfermería en Paraguay aún tiene una imagen profesional incipiente.

Y la verdad es que se hace lo que se puede [...] Yo creo que la parte de poder [...] enfermería [...] casi nada en el Paraguay [...] no se respeta más bien la parte de enfermería, no se le tiene en cuenta, muchas veces. Inclusive los familiares mismos, atropellan [...] Falta mucho [E2].

Hay quienes afirman que el empoderamiento existe cuando un enfermero asistencial es capaz de solucionar problemas durante su guardia sin supervisión y sin esperar que las autoridades reconozcan lo bueno de sus intervenciones.

Nosotros habitualmente solucionamos todo. Y... solucionamos por el bien del paciente $y$ nunca pedimos nada a cambio [E16].

Esta situación influye positivamente en el profesional de enfermería, quien luego de haber procurado una solución a eventuales inconvenientes, puede organizar el resto de las 
actividades durante su guardia. En segundo lugar se beneficia el paciente, quien recibe de parte del profesional los cuidados necesarios para su recuperación.

Otra idea importante recogida del discurso de los entrevistados, muestra que los profesionales reconocen que al no haber integración, trabajo en equipo y solidaridad, no se da empoderamiento profesional.

Si se considera las apreciaciones recogidas, la percepción de empoderamiento genera sentimientos encontrados que permiten afirmar que no existe empoderamiento profesional en el nivel individual.

\section{DISCUSIÓN}

Los resultados obtenidos muestran un grupo de profesionales jóvenes con predominio del sexo femenino y una permanencia entre 4 y 13 años en las unidades de cuidados intensivos. Estos datos coinciden con los encontrados en un estudio realizado por Rojas y Pastor $^{(5)}$ en las UCI de Medellín, Colombia, en donde las mujeres constituyen el $83 \%$ y el $75 \%$ de los encuestados reportó tener menos de 35 años. En relación al predominio del sexo femenino, este hallazgo también concuerda con lo descrito por García y Martínez ${ }^{(6)}$ al referirse a la enfermería como una profesión históricamente de mujeres.

En relación al tiempo que trabajan en el servicio, un estudio realizado por Pazetto y Kowal ${ }^{(7)}$ demuestra que las UCI constituyen un área de desempeño atractiva para los profesionales de enfermería, lo cual explica que la mayoría permanezca en ella por más de 5 años.

El sentido de pertenencia hacia la profesión de enfermería es incipiente. Esta motivación no alcanza a desarrollarse porque las relaciones y la convivencia no son del todo buenas, principalmente porque existen problemas no resueltos entre los enfermeros/as asistenciales. Otros factores que dificultan el desarrollo del sentido de pertenencia son la falta o pérdida de la integración de los miembros y la insatisfacción de algunos participantes por el trabajo que desempeñan en el servicio. Cabe resaltar que esta situación no es precisamente beneficiosa para los pacientes de los servicios críticos. Estos elementos fueron descritos por Ramírez, Perdomo y Galán ${ }^{(8)}$ como requisitos del profesional de enfermería

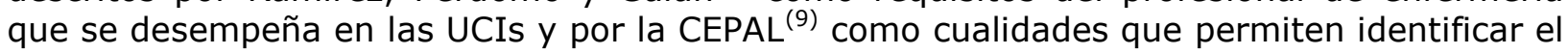
sentido de pertenencia.

Se evidencia autonomía en el ejercicio profesional, considerando que los profesionales de enfermería, toman decisiones clínicas relacionadas al cuidado del paciente. Los entrevistados manifiestan que la experiencia o práctica clínica y el conocimiento son elementos que determinan la competencia profesional, lo cual se ajusta a la teoría desarrollada por Patricia Benner ${ }^{(3)}$ en relación al desarrollo de la competencia profesional enfermera ${ }^{(10)}$. En un estudio realizado en Colombia por Tapp, Stansfield y Stewart ${ }^{(10)}$, se encontró que los enfermeros experimentan autonomía cuando pueden cumplir las metas de cuidado del paciente a través de sus conocimientos y habilidades.

En relación a los nueve dominios del profesional de enfermería de cuidados intensivos propuestos por Patricia Benner ${ }^{(3)}$ y que forman parte de la competencia profesional se encontraron presentes en los relatos de los participantes:

Dominio 1: diagnosticar y controlar las funciones fisiológicas de soporte vital en pacientes inestables.

Dominio 2: controlar una crisis mediante la actividad práctica.

Dominio 3: proporcionar medidas de comodidad a los pacientes en estado muy grave.

Dominio 5: prevenir los peligros por causas tecnológicas.

Dominio 7: comunicar las diferentes perspectivas y discutirlas.

Dominio 8: asegurar la calidad y solventar la crisis,

Dominio 9: poseer el conocimiento práctico hábil del liderazgo clínico y la formación y el consejo de los demás.

No se encontraron expresiones que muestren el dominio 4: cuidar a la familia del paciente y el dominio 6: afrontar la muerte, cuidados y toma de decisiones en pacientes terminales.

Un aspecto importante identificado en la literatura, es la implementación del Proceso de Atención de Enfermería (PAE) como herramienta que propicia la autonomía profesional. Este proceso no se utiliza en los servicios críticos del Hospital Central del IPS, lo cual demuestra que el reconocimiento de la autonomía no contempla el uso de PAE. 
El liderazgo constituye una práctica relativa entre los profesionales estudiados. Por una parte, identifican el rol de enfermero/a asistencial y reconocen sus funciones; por otra parte, el déficit actual de insumos, materiales y equipos constituye una limitación importante para la práctica del liderazgo. Esta realidad, aparte de perjudicar el ejercicio del liderazgo, se considera un factor que genera estrés en los enfermeros/as asistenciales de las $\mathrm{UCIs}^{(11)}$.

Según Guerrero y $\mathrm{Cid}^{(12)}$, el liderazgo es una cualidad que implica influir en las personas para el logro de los objetivos, permite el reconocimiento del grupo, la comunicación efectiva y la resolución oportuna de conflictos. Por su parte ${ }^{(12)}$, reconocen que influir en los demás, es fundamental para el ejercicio del liderazgo enfermero. Al respecto, este estudio evidencia debilidad en relación a la influencia positiva y por consecuencia, del liderazgo que debieran ejercer los enfermeros/as asistenciales dentro del equipo asistencial. Pazetto y Kowal( ${ }^{(7)}$ han encontrado similares resultados, al demostrar que la práctica del liderazgo no se relaciona con el entorno laboral de los enfermeros de cuidados intensivos; en este estudio los autores sugieren incorporar planes de desarrollo de líderes aptos para trabajar en el ámbito de cuidados intensivos.

El trabajo en equipo se define como una actividad coordinada en la cual las personas respetan sus roles y funciones para lograr objetivos comunes. Los profesionales estudiados perciben que esta cualidad es importante y destacan que se favorece cuando hay buena comunicación y respeto. Del Barrio y Reverte ${ }^{(13)}$ en un estudio realizado en UCIs de España, también reconocen que estas características favorecen el trabajo en equipo de los profesionales de enfermería.

Una inquietud importante, se verbaliza en la necesidad de una mayor formación en relaciones humanas. Esta demanda, también fue manifestada en el estudio realizado en Brasil por Pazetto y $\mathrm{Kowal}^{(7)}$; en este caso los participantes solicitaban más capacitación en el campo de las habilidades sociales.

Al examinar la participación que tienen los enfermeros/as en las asociaciones y sindicatos existentes, se encontró que en ambos casos la afiliación y la intervención son escasas y más bien corresponde a una necesidad de contar con respaldo para casos de emergencia. Este hallazgo contradice la propuesta de Zimmerman $^{(2)}$, quien sugiere que la participación en organizaciones, es uno de los factores que permite a las personas alcanzar empoderamiento individual $^{(10)}$.

Los enfermeros asistenciales perciben que el empoderamiento es una condición que permite tomar decisiones para solucionar problemas de forma creativa. En sus discursos, algunos participantes no identifican el significado de sentirse empoderado, sin embargo a través de sus relatos se evidencian atributos del empoderamiento. Morie, et al. ${ }^{(14)}$ muestran un resultado similar en un estudio realizado en México a profesionales de enfermería y estudiantes del último año de la carrera.

Al analizar los resultados obtenidos en la categoría percepción de empoderamiento desde la perspectiva teórica propuesta por Marc Zimmerman ${ }^{(2)}$, para el desarrollo del empoderamiento individual se observa:

-Que el aprendizaje de habilidades necesario durante el proceso de potenciación está presente. Los enfermeros asistenciales de las unidades de cuidados intensivos, manifiestan que el aprendizaje es permanente y en algunos casos, se han especializado en medicina crítica para desempeñarse eficientemente en el servicio. Se observa como resultado sentido de control en las actividades propias del cuidado del paciente crítico.

-Que el manejo de recursos se ve amenazado por la evidente escasez de insumos y equipos referida por los participantes de este estudio. Se observa como resultado conciencia crítica que permite distinguir lo perjudicial de esta situación.

-Que "trabajar con los demás" es una práctica habitual dentro de los servicios. Los participantes demuestran que trabajan en equipo tanto entre profesionales de enfermería como con los médicos del servicio de cuidados intensivos. Sólo excepcionales casos refieren conflictos de relacionamiento que atentan contra el trabajo en equipo. Se observa como resultado comportamiento participativo.

Otro hallazgo importante en la percepción de empoderamiento muestra que algunos participantes aún manifiestan dependencia y subordinación médica en la toma de decisiones lo cual demuestra ausencia de empoderamiento. Una situación similar describe Cadena(15) en su ensayo al afirmar que los profesionales de enfermería deben generar evidencia para 
desterrar de las indicaciones médicas la prescripción cuidados generales de enfermería lo cual anula las posibilidades de empoderamiento.

Es preocupante la percepción expresada por los participantes de este estudio, en relación a que los familiares no valoran las acciones de enfermería. Esta realidad disminuye la posibilidad de sentirse empoderado por cuanto existe estigmatización que subvalora la profesión, lo cual concuerda con lo expresado por Nieto ${ }^{(16)}$.

\section{REFERENCIAS BIBLIOGRÁFICAS}

1. Murguilday C. Reflexiones feministas sobre el empoderamiento de las mujeres. Colección cooperación [internet]. Barcelona: Cooperación; 2013 [acceso 22 de octubre de 2015]. Disponible en: http://www.cooperaccio.org

2. Zimmerman M. Empowerment theory: phycological, organizational and community levels of analisys. New York: Handbook of community psycology; 2000.

3. Benner P. From novice to expert: excellence and power in clinical nursing practice. Commemorative ed. New Jersey: Prentice-Hall Inc; 2001.

4. Burgos N. Investigación cualitativa. Buenos Aires: Espacio; 2011.

5. Rojas J, Pastor P. Aplicación del proceso de atención de enfermería en cuidados intensivos. Investigación y educación en enfermería. Invest Educ Enferm. 2010; 28(3): 323-35.

6. García C, Martínez M. Historia de la Enfermería: evolución histórica del cuidado enfermero. Madrid: Elsevier; 2001.

7. Pazetto A, Kowal I. Liderazgo en enfermería en las unidades de cuidados intensivos y su relación con el entorno laboral. Rev Latino Am Enfermagem 2015; 23 (1): 106-13.

8. Ramírez C, Perdomo A, Galán E. Evaluación de la calidad del cuidado de enfermería en la unidad de cuidados intensivos. Unal edu 2013; 31(1):42-51.

9. CEPAL. Cohesión social y sentido de pertenencia en América Latina y el Caribe. Santiago de Chile: CEPAL; 2007.

10. Tapp D, Stansfield K, Stewart J. La autonomía en la práctica de enfermería.
Aquichan 2005: 5(1). [acceso en fecha 26 de julio 2016]. Disponible en http://aquichan.unisabana.edu.co/index.php /aquichan/article/view/65

11. Menor M, Díaz R, Fernández L. Factores estresores y síndrome de Burnout en enfermeras de cuidados intensivos en dos hospitales de Salud nivel III-2 Lima Metropolitana. Cuidado y Salud. 2015; 13747.

12. Prado E, Cañete $M$. Reflexiones sobre el liderazgo en Enfermería. Revista VEA. 2010; 6(22):44-9.

13. Del Barrio $M$, Reverte $M$. Evaluación del trabajo en equipo en seis unidades de cuidados intensivos de dos hospitales universitarios. Trabajo presentado al curso de Administración y Gestión en Cuidados de Enfermería para la obtención del Master. Universitat de Barcelona; 2008.

14. Moriel-Corral L, Pizarro N, HernándezHernández $H$, Bonilla-Loyo E. Empoderamiento de enfermería como profesión y sujeto social: Caso Chihuahua. Rev Comun SEECI. 15 de marzo de 2012;0(27):30-8.

15. Cadena J. Análisis reflexivo del empoderamiento de la enfermería mexicana: mito o realidad. Medigraphic $2015 ; 23(1)$ :426.

16. Nieto R. Una revolución desde lo cotidiano. Empoderamiento Enfermero [internet] Granada: Enfermera 2.0; 20 de febrero de 2012 [acceso 2 de marzo de 2016]. Disponible en: http://enfermeradospuntocero.blogspot.com 